\section{Singular structures and operon organizations of essential two-component systems in species of Streptococcus}

Two component regulatory systems (TCSs) are found in all prokaryotes except mycoplasmas (Fraser et al., 1995; Himmelreich et al., 1996). They play critical roles in sensing and responding to environmental conditions and in bacterial pathogenesis (Hoch, 2000; Inouye \& Dutta, 2003; Stock et al., 2000). TCSs consist of a sensor histidine kinase protein, which is usually bound to the bacterial membrane (Wolanin \& Stock, 2003), and a cognate response regulator protein, which often acts as a DNA-binding transcription regulator. Autophosphorylation of the histidine kinase component in response to an environmental signal is followed by phosphoryl transfer to the cognate response regulator, which alters the expression of regulon genes needed to respond to the environmental condition.

Most TCSs are not required for bacteria to grow in the absence of stress in laboratory media (Kobayashi et al., 2001; Oshima et al., 2002). However, there are exceptions to this generalization in certain Gram-negative bacteria [e.g. Caulobacter crescentus (Quon et al., 1996)] and in all Gram-positive bacteria with low $\mathrm{G}+\mathrm{C}$ content in their DNA. Each of these
Gram-positive species, which include many important human pathogens (see Table 1), contains homologues of a single essential TCS, whose histidine kinase and response regulator are designated $\mathrm{YycG}$ and $\mathrm{YycF}$, respectively. The gene encoding the $\mathrm{YycF}$ response regulator is required for growth of these Gram-positive bacteria under all conditions tested (Echenique \& Trombe, 2001; Fabret \& Hoch, 1998; Martin et al., 1999; Ng et al., 2003). The YycG histidine kinase is required for growth of most Gram-positive species tested to date (Fabret \& Hoch, 1998; Martin et al., 1999) or is conditionally required in Streptococcus pneumoniae depleted for the $\mathrm{YycF}$ response regulator (Ng et al., 2003).

The genes regulated and signal(s) sensed by these essential TCSs in different

Table 1. Characteristics of YycG histidine kinases from Gram-positive bacteria with fully sequenced genomes

\begin{tabular}{|c|c|c|c|c|}
\hline Species & YycG homologue ${ }^{\star}$ & $\begin{array}{c}\text { Number of predicted } \\
\text { TM domains } \dagger\end{array}$ & $\begin{array}{l}\text { Presence of YycH and } \\
\text { YycI homologues } \ddagger\end{array}$ & $\begin{array}{c}\text { Capacity for } \\
\text { electron transport } \$\end{array}$ \\
\hline Streptococcus pneumoniae & Spr1106, SP1226 & 1 & No & No \\
\hline Streptococcus mutans & Smu.1516 & 1 & No & No \\
\hline Streptococcus agalactiae & SAG0720, Gbs0742 & 1 & No & Yes \\
\hline Enterococcus faecalis & EF1194 & $2(142)$ & Yes & Yes \\
\hline Lactobacillus plantarum & Lp_0037 & $2(147)$ & Yes & Yes \\
\hline Lactobacillus johnsonii & LJ0066 & $2(142)$ & Yes & Yes \\
\hline Lactococcus lactis & L0124 & $2(4)$ & No & Yes \\
\hline Listeria innocua & Lin0316 & $2(148)$ & Yes & Yes \\
\hline Bacillus anthracis & BA5714 & $2(154)$ & Yes & Yes \\
\hline Bacillus subtilis & Bsu4037 & $2(148)$ & Yes & Yes \\
\hline Staphylococcus epidermidis & SE0019 & $2(147)$ & Yes & Yes \\
\hline Staphylococcus aureus & SA0018, SAV0019, MW0019 & $2(145)$ & Yes & Yes \\
\hline Bacillus halodurans & BH4026 & $2(146)$ & Yes & Yes \\
\hline Oceanobacillus iheyensis & OB3451 & $2(154)$ & Yes & Yes \\
\hline
\end{tabular}

${ }^{*}$ YycG homologues were identified by BLAST searches of each complete genome sequence using B. subtilis yycG as the query. Only the match with highest homology in each genome was analysed. Multiple listings represent homologues in different strains of the same species.

$\dagger$ Number of transmembrane (TM) domains was predicted by the TMHMM program (Krogh et al., 2001). A single TM domain, first noted in the YycG homologue of S. pneumoniae (Lange et al., 1999) (see Fig. 1b), is present in the YycG homologues of all Streptococcus species (see text). Numbers in parentheses indicate the number of amino acid residues in predicted extracytoplasmic loops of YycG homologues with two TM domains (see Fig. 1a).

$\ddagger B$. subtilis $y y c H$ and $y y c I$ were used as queries to search for homologues in each completed genome. Homologues of $B$. subtilis $y y c J$ are present in all genomes analysed (see text).

\$Capacity for electron transport based on the presence of at least one homologue of the following B. subtilis genes encoding aerobic terminal oxidases (QoxABCD, CtaCDEF, CydAB and YthAB) (von Wachenfeldt \& Hederstedt, 2002) or anaerobic nitrate reductase (NarGHIJ) (Hoffmann et al., 1995). 
Gram-positive species are of considerable interest. Recent results from several laboratories indicate that the regulons controlled by YycFG TCSs seem to be different in different Gram-positive bacteria (Dubrac \& Msadek, 2004; Fukuchi et al., 2000; Howell et al., 2003; Ng et al., 2003). However, the pattern emerging suggests that YycFG controls genes critical for cell wall biosynthesis, cell division and virulence (Dubrac \& Msadek, 2004; Fukuchi et al., 2000; Howell et al., 2003; Ng et al., 2003, 2004). The signal(s) sensed by the YycG histidine kinase homologues are not known (Claverys \& Havarstein, 2002; Dubrac \& Msadek, 2004; Fukuchi et al., 2000; Howell et al., 2003; Ng et al., 2003). We describe here a potentially important structural difference in the $\mathrm{YycG}$ homologues and the operons that encode them for species of Streptococcus compared to those of other Gram-positive bacteria.

\section{All YycG homologues contain a HAMP} (linker) domain, a PAS domain, a histidine kinase domain (HisKin, which contains the phosphorylated histidine residue) and an ATPase domain (Fig. 1; Aravind \& Ponting, 1999; Inouye \& Dutta, 2003; Taylor \& Zhulin, 1999). PAS domains of histidine kinases bind cofactors that respond to redox state (Taylor \& Zhulin, 1999) and also act as sites of protein-protein interactions (Elsen et al., 2003; Taylor \& Zhulin, 1999; Wang et al.,
2001). Most histidine kinases, including YycG homologues from all Gram-positive species except for Streptococcus, are likely bound to the cytoplasmic membrane by two transmembrane domains that flank an extracytoplasmic loop (Fig. 1a). These extracytoplasmic loops are thought to play roles in sensing by some histidine kinases (Hoch, 2000; Wolanin \& Stock, 2003).

The extracytoplasmic loops of these $\mathrm{YycG}$ homologues are generally large and contain 142-154 amino acids (Table 1). In contrast, the YycG homologues of Streptococcus species contain a single predicted transmembrane domain (Fig. 1b), and hence lack extracytoplasmic loops (Table 1). The number of extracellular amino acids that protrude from these single transmembrane domains is small (4-12 amino acids; Fig. 1b; Table 1).

We noticed another structural difference between the YycFG TCS homologues of Streptococcus and those of other Gram-positive species. The genes encoding the YycFG two-component system are cotranscribed in all Gram-positive bacteria with another gene, designated $y y c J$ (Fukuchi et al., 2000; Wagner et al., 2002). The YycJ protein contains a predicted $\mathrm{HxHxDH}$ metal-binding site in a $\beta$-lactamase fold (Daiyasu et al., 2001). This type of metal-binding site binds zinc or iron ions in enzymes with different activities (Daiyasu et al., 2001). The

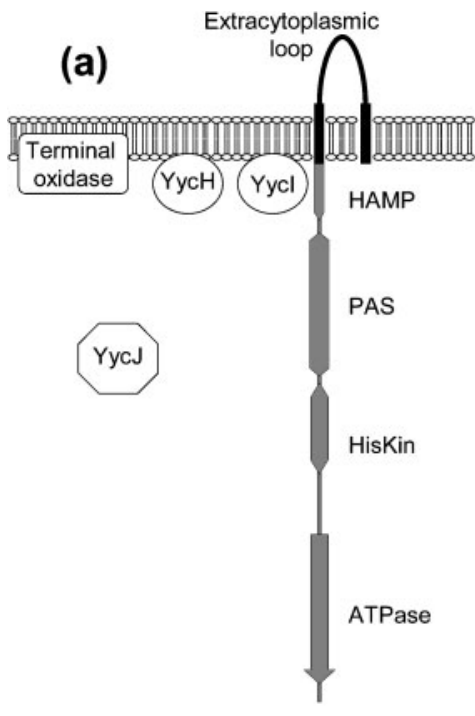

YycG in most Gram-positives

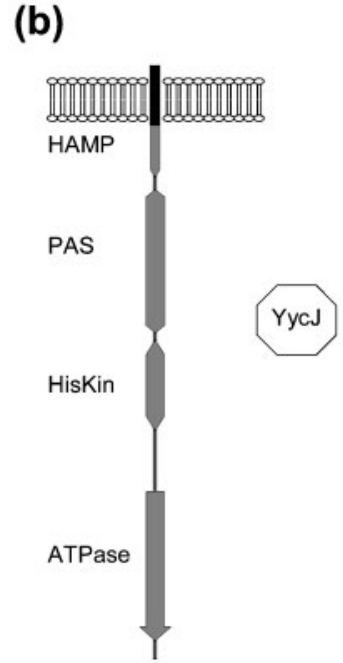

YycG in Streptococcus species

Fig. 1. Structural motifs in YycG histidine kinase homologues. See text for details.

function(s) of YycJ is unknown, although the YycJ homologue of S. pneumoniae is conditionally essential for growth in bacteria limited for expression of the $\mathrm{YycF}$ response regulator ( $\mathrm{Ng}$ et al., 2003). However, in Gram-positive species other than Streptococcus, $y y c F G$ and $y y c J$ are cotranscribed with two additional genes, designated $y y c H$ and $y y c I$, which encode proteins containing transmembrane domains (Fabret \& Hoch, 1998; Fukuchi et al., 2000). With one exception, Gram-positive species that encode $\mathrm{YycH}$ and YycI homologues contain YycG homologues with two transmembrane domains flanking extracytoplasmic loops (Fig. 1a; Table 1). The exception here is Lactococcus lactis, which encodes a YycG homologue with two transmembrane domains, but lacks $y y c H$ and $y y c I$ genes (Table 1). However, the L. lactis YycG homologue is itself unusual in that it contains a predicted extracytoplasmic loop of just 4 amino acids, which is much smaller than those of other YycG homologues (Fig. 1a).

The structural and operon differences of the YycFG TCSs of Streptococcus species compared to those of other Gram-positive species suggest important differences in signal transduction. There are clearly two distinct classes of YycG histidine kinases (Fig. 1; Table 1), which tends to argue against the view that the extracytoplasmic domains of YycG homologues became randomized during evolution (Hoch, 2000). Moreover, a different subset of associated components (YycJ, $\mathrm{YycH}$ and $\mathrm{YycI}$ ) is associated with each $\mathrm{YycG}$ class (Table 1). These structural differences imply that different signals or subsets of signals may be sensed by YycG homologues in Streptococcus species compared to those sensed in other Gram-positive species. It is noteworthy that Gram-positive species containing YycG histidine kinases with two transmembrane domains flanking large extracytoplasmic loops (Fig. 1a) and associated $\mathrm{YycH}$ and $\mathrm{YycI}$ components have the capacity for electron transport, which is absent from all species of Streptococcus, except for Streptococcus agalactiae (Table 1). Perhaps, the extended extracytoplasmic domain of YycG and the $\mathrm{YycH}$ and $\mathrm{YycI}$ components sense the redox state of electron transport. 
On the other hand, the cytoplasmic PAS domains of all YycG homologues and the associated YycJ components may sense small molecule and cofactor signals that reflect metabolic state, such as those that accumulate during aerobic or anaerobic growth or stress (Nakano \& Zuber, 1998; Taylor \& Zhulin, 1999). The metabolisms and physiological niches of different Gram-positive bacteria vary considerably. These differences may be reflected by the different sets of genes regulated by the YycFG TCSs (Dubrac \& Msadek, 2004; Fukuchi et al., 2000; Howell et al., 2003; $\mathrm{Ng}$ et al., 2003). It remains to be determined whether the same or different small molecules' signals are sensed by $\mathrm{YycG}$ homologues in these disparate species.

\section{Acknowledgements}

Supported by research funds from Indiana University Bloomington. Wai-Leung $\mathrm{Ng}$ is a predoctoral trainee on grant NIGM-T32GM0775 from the National Institutes of Health of the USA.

\section{Wai-Leung $\mathrm{Ng}$ and Malcolm E. Winkler}

Department of Biology, Indiana University, Bloomington, IN 47405, USA

\section{Correspondence: Malcolm E. Winkler} (mwinkler@bio.indiana.edu)

Aravind, L. \& Ponting, C. P. (1999). The cytoplasmic helical linker domain of receptor histidine kinase and methyl-accepting proteins is common to many prokaryotic signalling proteins. FEMS Microbiol Lett 176, 111-116.

Claverys, J. P. \& Havarstein, L. S. (2002). Extracellular-peptide control of competence for genetic transformation in Streptococcus pneumoniae. Front Biosci 7, d1798-d1814.

Daiyasu, H., Osaka, K., Ishino, Y. \& Toh, H. (2001). Expansion of the zinc metallo-hydrolase family of the beta-lactamase fold. FEBS Lett 503, 1-6.

Dubrac, S. \& Msadek, T. (2004). Identification of genes controlled by the essential YycG/YycF two-component system of Staphylococcus aureus. J Bacteriol 186, 1175-1181.

Echenique, J. R. \& Trombe, M. C. (2001). Competence repression under oxygen limitation through the two-component MicAB signal-transducing system in Streptococcus pneumoniae and involvement of the PAS domain of MicB. J Bacteriol 183, $4599-4608$.
Elsen, S., Duche, O. \& Colbeau, A. (2003). Interaction between the $\mathrm{H} 2$ sensor HupUV and the histidine kinase HupT controls HupSL hydrogenase synthesis in Rhodobacter capsulatus. J Bacteriol 185, 7111-7119.

Fabret, C. \& Hoch, J. A. (1998). A two-component signal transduction system essential for growth of Bacillus subtilis: implications for anti-infective therapy. J Bacteriol 180, 6375-6383.

Fraser, C. M., Gocayne, J. D., White, O. \& 26 other authors (1995). The minimal gene complement of Mycoplasma genitalium. Science 270, 397-403.

Fukuchi, K., Kasahara, Y., Asai, K., Kobayashi, K., Moriya, S. \& Ogasawara, N. (2000). The essential two-component regulatory system encoded by $y y c F$ and $y y c G$ modulates expression of the fts $A Z$ operon in Bacillus subtilis. Microbiology 146, 1573-1583.

Himmelreich, R., Hilbert, H., Plagens, H., Pirkl, E., Li, B. C. \& Herrmann, R. (1996). Complete sequence analysis of the genome of the bacterium Mycoplasma pneumoniae. Nucleic Acids Res 24, 4420-4449.

Hoch, J. A. (2000). Two-component and phosphorelay signal transduction. Curr Opin Microbiol 3, 165-170.

Hoffmann, T., Troup, B., Szabo, A., Hungerer, C. \& Jahn, D. (1995). The anaerobic life of Bacillus subtilis: cloning of the genes encoding the respiratory nitrate reductase system. FEMS Microbiol Lett 131, 219-225.

Howell, A., Dubrac, S., Andersen, K. K., Noone, D., Fert, J., Msadek, T. \& Devine, K. (2003). Genes controlled by the essential YycG/YycF two-component system of Bacillus subtilis revealed through a novel hybrid regulator approach. Mol Microbiol 49, 1639-1655.

Inouye, M. \& Dutta, R. (2003). Histidine Kinases in Signal Transduction. San Diego, CA: Academic Press.

Kobayashi, K., Ogura, M., Yamaguchi, H., Yoshida, K., Ogasawara, N., Tanaka, T. \& Fujita, Y. (2001). Comprehensive DNA microarray analysis of Bacillus subtilis two-component regulatory systems. J Bacteriol 183, 7365-7370.

Krogh, A., Larsson, B., von Heijne, G. \& Sonnhammer, E. L. (2001). Predicting transmembrane protein topology with a hidden Markov model: application to complete genomes. J Mol Biol 305, 567-580.

Lange, R., Wagner, C., de Saizieu, A. \& 7 other authors (1999). Domain organization and molecular characterization of 13 twocomponent systems identified by genome sequencing of Streptococcus pneumoniae. Gene 237, 223-234.

Martin, P. K., Li, T., Sun, D., Biek, D. P. \& Schmid, M. B. (1999). Role in cell permeability of an essential two-component system in Staphylococcus aureus. J Bacteriol 181, 3666-3673.

Nakano, M. M. \& Zuber, P. (1998). Anaerobic growth of a "strict aerobe" (Bacillus subtilis). Annu Rev Microbiol 52, 165-190.

Ng, W. L., Robertson, G. T., Kazmierczak, K. M., Zhao, J., Gilmour, R. \& Winkler, M. E. (2003). Constitutive expression of PcsB suppresses the requirement for the essential VicR (YycF) response regulator in Streptococcus pneumoniae R6. Mol Microbiol 50, 1647-1663.

Ng, W. L., Kazmierczak, K. M. \& Winkler, M. E. (2004). Defective cell wall synthesis in Streptococcus pneumoniae R6 depleted for the essential PcsB putative murein hydrolase or the VicR (YycF) response regulator. Mol Microbiol 53, 1161-1175.

Oshima, T., Aiba, H., Masuda, Y., Kanaya, S., Sugiura, M., Wanner, B. L., Mori, H. \& Mizuno, T. (2002). Transcriptome analysis of all two-component regulatory system mutants of Escherichia coli K-12. Mol Microbiol 46, 281-291.

Quon, K. C., Marczynski, G. T. \& Shapiro, L. (1996). Cell cycle control by an essential bacterial two-component signal transduction protein. Cell 84, 83-93.

Stock, A. M., Robinson, V. L. \& Goudreau, P. N. (2000). Two-component signal transduction. Annu Rev Biochem 69, 183-215.

Taylor, B. L. \& Zhulin, I. B. (1999). PAS domains: internal sensors of oxygen, redox potential, and light. Microbiol Mol Biol Rev 63, 479-506.

von Wachenfeldt, C. \& Hederstedt, L. (2002). Respiratory cytochromes, other heme proteins, and heme biosynthesis. In Bacillus subtilis and Its Closest Relatives: From Gene to Cells,

pp. 163-179. Edited by A. L. Sonenshein, J. A. Hoch \& R. Losick. Washington, DC: American Society for Microbiology.

Wagner, C., Saizieu Ad, A., Schonfeld, H. J., Kamber, M., Lange, R., Thompson, C. J. \& Page, M. G. (2002). Genetic analysis and functional characterization of the Streptococcus pneumoniae vic operon. Infect Immun 70, 6121-6128.

Wang, L., Fabret, C., Kanamaru, K., Stephenson, K., Dartois, V., Perego, M. \& Hoch, J. A. (2001). Dissection of the functional and structural domains of phosphorelay histidine kinase A of Bacillus subtilis. J Bacteriol 183, 2795-2802.

Wolanin, P. M. \& Stock, J. B. (2003).

Transmembrane signaling and the regulation of histidine kinase activity. In Histidine Kinases in Signal Transduction, pp. 74-123. Edited by M. Inouye \& R. Dutta. Academic Press.

DOI 10.1099/mic.0.27550-0 